\title{
MODELAGEM MATEMÁTICA DA PREDIÇÃO DO TEMPO DE VIDA DE UMA BATERIA UTILIZANDO A ESTRUTURA DE MODELO AUTORREGRESSIVO DA TEORIA DA IDENTIFICAÇÃO DE SISTEMAS
}

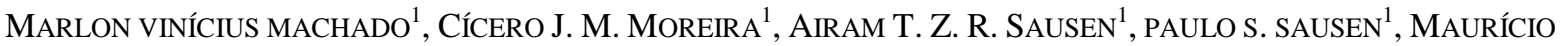 \\ DE CAMPOS ${ }^{1}$
}

\author{
1. Programa de Pós-Graduação Stricto Sensu, Departamento de Ciências Exatas e Engenharias, Univer- \\ sidade Regional do Noroeste do Estado do Rio Grande do Sul, Unijuí \\ Rua do Comércio, 3000 - Campus Ijuí - RS - Brasil \\ E-mails: mmmat15@hotmail.com, cicero.matematica@gmail.com, ai- \\ ram@unijui.edu.br, sausen@unijui.edu.br, campos@unijui.edu.br
}

\begin{abstract}
Portable Electronic Systems are increasingly being sought by the company, either for leisure or for work. And this happens due to ease these devices bring to everyday life, and by their mobility, easy access to wireless networks, size, weight, functionality, among other. However, for their operation, portable electronic systems require a power source, the battery. Thus, this study seeks to find in the Theory of Identification Systems, a mathematical model to make the prediction of the lifetime of a battery. For this we conducted a literature review on batteries, on the state of the art of mathematical modeling to predict the lifetime of batteries and the Theory of System Identification. Later, was chosen a structure model of the Theory of System Identification, Auto Regressive model. Met the parameters of the model that fits the experimental data set obtained from a test platform, built for this purpose. The computational model was implemented using the software tool MATLAB and then held validation. The study with the Auto Regressive model showed good results.
\end{abstract}

Keywords- Portable Electronic Systems, Battery Life Time, Auto-Regressive Model, System Identification, Mathematics Modelling.

\begin{abstract}
Resumo- Os dispositivos móveis estão sendo cada vez mais procurados pela sociedade, seja para o lazer ou para o trabalho. E isto se dá devida facilidade que estes aparelhos trazem ao cotidiano, sendo por sua mobilidade, fácil acesso as redes sem fio, tamanho, peso, funcionalidade, entre outras. Porém, para o seu funcionamento, os dispositivos móveis necessitam de uma fonte de energia, a bateria. Sendo assim, este estudo busca encontrar na Teoria da Identificação de Sistemas, um modelo matemático para realizar a predição do tempo de vida de uma bateria. Para isto foi realizada uma revisão bibliográfica sobre baterias, sobre o estado da arte da modelagem matemática na predição do tempo de vida de baterias e sobre a Teoria da Identificação de Sistemas. Posteriormente, foi escolhida uma estrutura de modelo da Teoria da Identificação de Sistemas, modelo Autorregressivo. Encontrou-se os parâmetros do modelo que se adapta ao conjunto de dados experimentais obtidos de uma plataforma de testes, construída para esta finalidade. Este modelo foi implementado computacionalmente usando a ferramenta computacional MATLAB e, posteriormente, realizada sua validação. O estudo com o modelo Autorregressivo mostrou bons resultados.
\end{abstract}

Palavras-chave— Dispositivos Móveis, Tempo de vida de baterias, Modelo Autorregressivo, Identificação de Sistemas, Modelagem Matemática.

\section{Introdução}

Nas últimas décadas, o número médio de dispositivos móveis por cidadão tem aumentado significativamente devido, principalmente, à proliferação no acesso à tecnologia sem fio e a sua mobilidade. Estes dispositivos podem ser encontrados nas mais diversas áreas, tais como, na indústria, nos setores de educação e saúde, sendo utilizados também no lazer e no entretenimento. Alguns exemplos de dispositivos móveis são: telefones celulares, máquinas digitais, notebooks (computadores portáteis), smartphones, tablets, sensores de alarmes instalados em residências ou prédios comerciais, entre outros.

A grande maioria dos dispositivos móveis, utilizados atualmente, é alimentada por algum tipo de bateria, geralmente recarregável, cuja função é o fornecimento de energia ao sistema. Destaca-se que a utilização destes dispositivos está condicionada ao tempo de vida das baterias que os alimentam, que é por definição, o tempo que a bateria demora até atingir um determinado nível de capacidade de carga (i.e., nível de cutoff). Ao alcançar este nível as reações eletroquímicas, que são responsáveis pela gera- ção de energia, deixam de acontecer e, então, a bateria pára de fornecer energia ao sistema. Neste contexto, é de vital importância possuir algum método capaz de predizer o tempo de vida da bateria e consequentemente do dispositivo que é alimentado por ela.

Existem diferentes maneiras de realizar a predição do tempo de vida de baterias, uma delas consta da experimentação física. Porém, dependendo das características do sistema, esta opção deixa de ser viável do ponto de vista econômico. Outra forma é utilizando modelos matemáticos que representem a descarga de energia do sistema. Dentre os modelos matemáticos de baterias, podem ser citados: os analíticos (Lahiri et. al. (2002), Martin (1999), Rakhmatov e Vrudhula (2001), Sausen (2008)), os estocásticos (Chiasserini e Rao (1999)), os elétricos (Lahiri et. al. (2002)) e os eletroquímicos (Chen e RincónMora (2006)), cada um com suas características e níveis de complexidade.

Na literatura técnica os modelos analíticos são apresentados como os modelos mais fáceis de serem usados, se comparados com os modelos elétricos e os eletroquímicos, pois descrevem as propriedades da 
bateria através de simples expressões analíticas (Jongerden e Haverkort (2008)), são considerados, ainda, mais flexíveis, de fácil e boa eficiência computacional (Lahiri et. al. 2002). Tendo em vista isto, Oliveira (2012) realizou um estudo sobre alguns modelos analíticos, objetivando verificar qual o melhor para realizar a predição do tempo de vida de baterias. Os modelos analíticos estudados foram o Modelo Linear, a Lei de Peukert e o Modelo de Difusão de Rakhmatov e Vrudhula, os quais mostraram uma precisão de $82,58 \%, 92,9 \%$, e $94,29 \%$ respectivamente, onde verificou que o Modelo de Difusão de Rakhmatov-Vrudhula, foi o mais preciso, com um erro médio de 5,71\%. Romio (2013), em seus estudos, também utilizou o modelo de Difusão Rakhmatov-Vrudhula considerando um conjunto de dados diferente do trabalho proposto por Oliveira (2012), encontrando um erro médio de 5,68\%.

Estudos recentes relacionados à modelagem matemática de sistemas reais mostram uma mudança, a partir da década de 90, que aponta para uma crescente necessidade de desenvolvimento de modelos matemáticos a partir de dados observados. Esta mudança tem ocorrido por vários motivos, dentre eles é possível destacar que os sistemas utilizados, com o desenvolvimento da ciência/tecnologia, estão cada vez mais complexos ocasionando, muitas vezes, até a impossibilidade de escrever as equações básicas que representam o comportamento fenomenológico do mesmo através da física do processo. Também, observa-se que os computadores tornaram-se mais acessíveis do ponto de vista financeiro e de desempenho, viabilizando o seu maior uso no processamento de dados coletados diretamente das plantas e, a partir de tais observações torna-se possível desenvolver/aplicar equações de modelos que são capazes de explicar tais dados. Esta modelagem é conhecida como Identificação de Sistemas.

Segundo Aguirre (2007), a teoria de Identificação de Sistemas propõe encontrar um modelo matemático que, aproximadamente, represente e explique a relação causa e efeito presente em um conjunto de dados. Neste contexto, o principal objetivo deste artigo é a obtenção de um modelo matemático acurado e de simples implementação para a predição do tempo de vida de baterias utilizadas em dispositivos móveis usando a estrutura de modelo do tipo AutoRegressivo (AR), presente na teoria de Identificação de Sistemas. Em seguida o modelo obtido será comparado ao Modelo de Difusão de RakhmatovVrudhula, que é o modelo analítico mais acurado da literatura técnica. As baterias usadas nos processos de descarga são do tipo Lithium-íon, modelo Nokia BL-5F, presentes em celulares Nokia N95.

O restante deste artigo está organizado como segue. Na Seção 2 é apresentada a estrutura do modelo matemático AR. Na Seção 3 são apresentados os métodos para a obtenção do modelo AR. Na Seção 4 são apresentados os resultados das simulações e análise. Na Seção 5 são apresentadas as conclusões.

\section{Modelo Matemático}

Segundo Morettin (1987) o modelo AR é um exemplo de modelo univariado de série temporal em que uma variável aleatória está relacionada com os próprios valores passados e com os erros aleatórios. Ou seja, considerando o sistema dinâmico, ao se utilizar de um modelo AR, se está comparando a saída no instante $n$ com as saídas nos instantes $(n-1),(n-2)$, e assim por diante. E este tipo de modelo é representado pela equação dada por:

$$
A(q) y(k)=v(k) \Rightarrow y(k)=\frac{1}{A(q)} v(k)
$$

onde: $A(q)=1-a_{1} q^{-1}-\cdots-a_{n_{y}} q^{-n_{y}}$, é o polinômio de regressores; $q^{-1}$ é o operador de atraso; $y(k)$ são as saídas do sistema; $v(k)$ é o ruído branco.

\section{Métodos}

Para desenvolvimento deste estudo, primeiramente foram obtidos dados experimentais da descarga $(I)$ e do tempo de vida (TV) de uma bateria, através de uma plataforma de testes desenvolvida especificamente para esta finalidade, baseado em diferentes perfis de descargas, aplicados ao sistema. Esta plataforma foi desenvolvida no laboratório do Grupo de Automação Industrial e Controle (GAIC) da Universidade Regional do Noroeste do Estado do Rio Grande do Sul (UNIJUÍ).

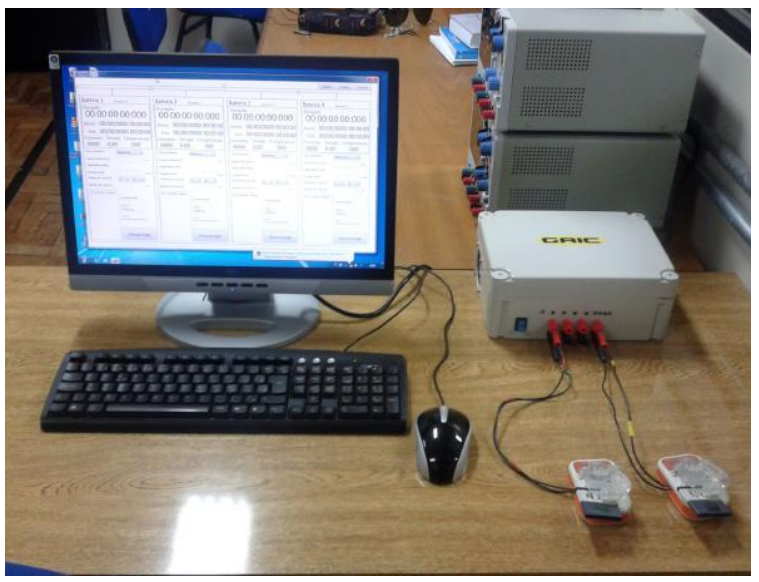

Figura 1. Plataforma de testes.

Este ambiente de testes, apresentado na Figura 1, é composto por um sistema de descargas controladas, o qual possibilita escolher qual o perfil de descarga que se quer aplicar na bateria. Este sistema de descarga está ligado a um microcomputador, que possui uma ferramenta de gerenciamento da plataforma que permite tanto administrar as descargas, quanto armazenar as informações obtidas do processo de descarga da bateria.

A metodologia adotada para a coleta de dados a partir da plataforma é descrita a seguir. Considera-se 
inicialmente a bateria completamente carregada (i.e., com uma voltagem de 4,2 volts), os experimentos foram divididos em dois momentos, o primeiro com dez perfis de descargas constantes utilizado para estimação dos parâmetros do modelo AR; e o segundo com seis perfis de descargas constantes usado para a validação do modelo AR. Para cada perfil de descarga considerado, os ensaios foram repetidos dez vezes, objetivando a obtenção de uma amostragem estatística satisfatória.

Tabela 1. Dados obtidos da plataforma de testes para a estimação dos parâmetros do modelo AR.

\begin{tabular}{|c|c|}
\hline$I(\mathrm{~mA})$ & $T V_{m}(\mathrm{~min})$ \\
\hline 150 & 194,84 \\
\hline 250 & 114,43 \\
\hline 350 & 77,95 \\
\hline 450 & 59,97 \\
\hline 550 & 48,46 \\
\hline 650 & 40,55 \\
\hline 750 & 36,12 \\
\hline 850 & 31,10 \\
\hline 950 & 26,08 \\
\hline
\end{tabular}

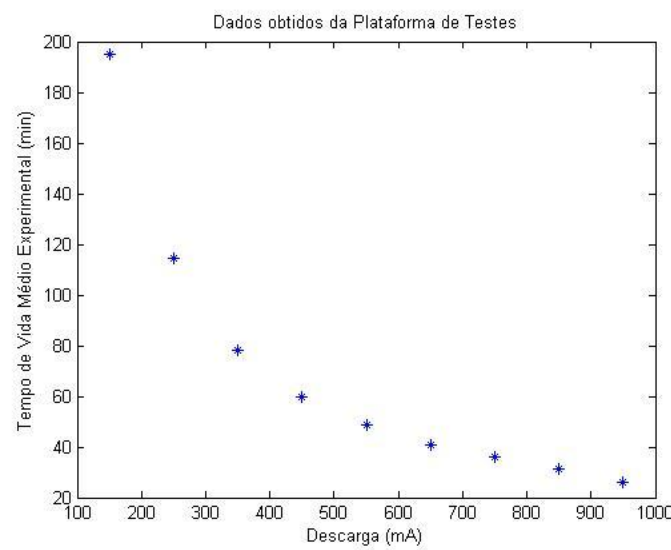

Figura 2. Dados Obtidos da Plataforma de Testes.

Tabela 2. Dados obtidos da plataforma de testes para validação do modelo AR.

\begin{tabular}{|c|c|}
\hline$I(\mathrm{~mA})$ & $T V_{m}(\mathrm{~min})$ \\
\hline 200 & 136,66 \\
\hline 350 & 77,95 \\
\hline 500 & 49,38 \\
\hline 650 & 40,55 \\
\hline 800 & 28,8 \\
\hline 950 & 26,08 \\
\hline
\end{tabular}

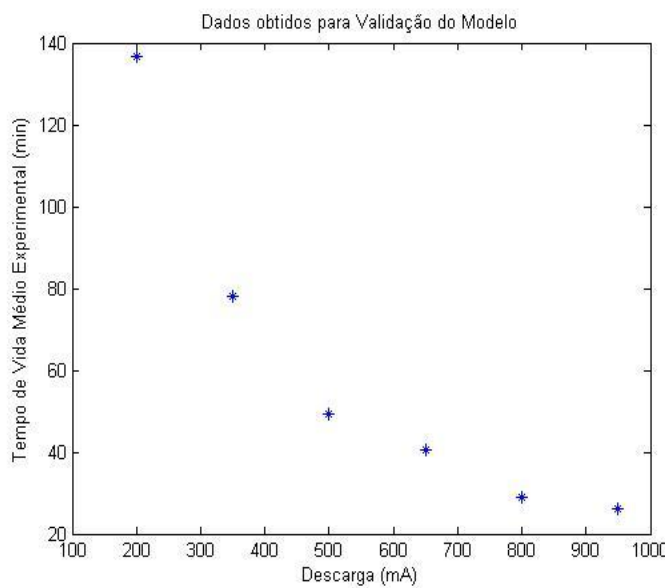

Figura 3. Dados obtidos para Validação do Modelo

Na Tabela 1 e na Figura 2, são apresentados os dados utilizados para a estimação dos parâmetros do modelo AR. Segundo Aguirre (2007) não se deve utilizar o mesmo conjunto de dados para a estimação dos parâmetros do modelo e para a sua validação, sendo assim, buscou-se um novo conjunto de dados utilizado somente para validação do modelo AR apresentados na Tabela 2 e na Figura 3. Observa-se que na primeira coluna de ambas as tabelas são apresentados os perfis de descarga $(I)$, e na segunda coluna o tempo de vida médio $\left(T V_{\mathrm{m}}\right)$.

Em paralelo com a obtenção dos dados de descarga da bateria, sua análise e apresentação, realizouse uma revisão bibliográfica sobre a teoria da Identificação de Sistemas e os modelos matemáticos que a compõem, a partir da qual, foi possível realizar a escolha da estrutura de modelo para realização deste estudo. A estrutura de modelo AR foi escolhida, por se mostrar mais simples e de fácil compreensão e implementação computacional. O modelo foi implementado utilizando a ferramenta computacional Matlab.

\section{Resultado das Simulações e Análise}

No modelo AR, definido na equação (1), quando se substitui o polinômio $A(q)$ é obtida a equação a diferenças dada por:

$$
y(k)=\theta_{1} \cdot y(k-1)+\theta_{2} \cdot y(k-2)
$$

onde os parâmetros $\theta_{1}$ e $\theta_{2}$ devem ser estimados.

Os parâmetros $\theta_{1}$ e $\theta_{2}$ foram estimados utilizando o método dos Mínimos Quadrados (MQ) (Anton e Rorres (2001)) e os dados da Tabela 1, onde os valores encontrados para cada parâmetro foram: $\theta_{1}=$ 1,2537 e $\theta_{2}=-0,3353$. Assim, o modelo AR apresentado na equação (2) torna-se:

$$
y(k)=1,2537 \cdot y(k-1)-0,3353 \cdot y(k-2)
$$


Em seguida o modelo AR foi validado considerando os dados da Tabela 2. Na Figura 5 é apresentada a validação do modelo AR .

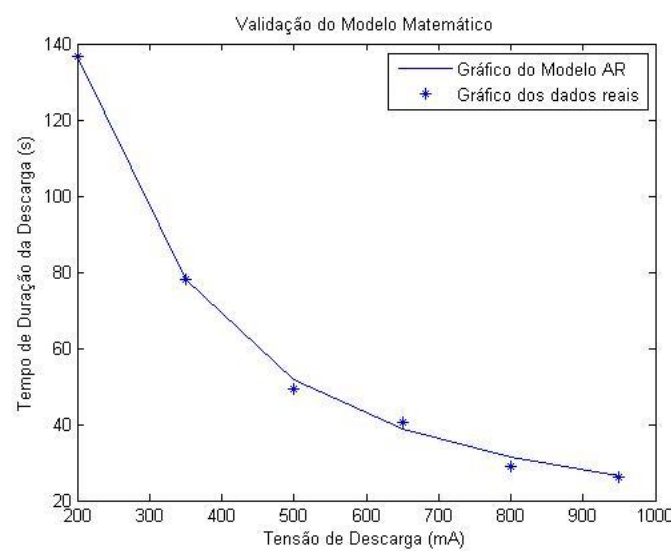

Figura 5 - Validação do Modelo Matemático.

Na Figura 6 é apresentado o gráfico do erro médio gerado pela comparação entre os dados calculados pelo modelo apresentado na equação (2) e os dados reais da Tabela 2. Este erro foi obtido a partir do Erro Relativo Percentual - RMSE.

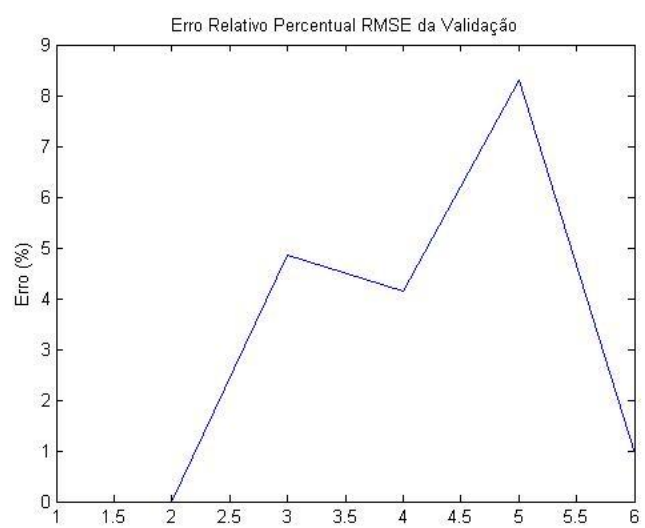

Figura 6 - Erro percentual

O erro médio encontrado na validação do modelo foi de aproximadamente $3,04 \%$, sendo assim, sua precisão foi de $96,96 \%$.

Em seus estudos, Romio (2013) utiliza do mesmo conjunto de dados, da Tabela 2, para validação dos modelos por ele estudados e, também, para comparação com o modelo de Difusão de Rakhmatov e Vrudhula. Sendo assim, devido a este ser o modelo mais acurado apresentado pela literatura técnica, os resultados obtidos por Romio (2013) para o Modelo de Difusão de Rakhmatov e Vrudhula, são utilizados neste trabalho para a comparação com os resultados do Modelo AR. Esta comparação se encontra na Tabela 3, onde $I$ representa os perfis de descargas da bateria, em $m A ; T V_{m}$ é o tempo de vida médio, em min, obtidos da plataforma de testes; $T V_{S A R}$ é o tempo de vida, em min, obtido pela simulação do modelo AR; $E_{A R}$ é o erro gerado pela simulação do modelo AR em comparação com os dados obtidos da plata- forma de testes, em \%; $T V_{S R V}$ é o tempo de vida, em min, obtido pela simulação do modelo de Difusão de Rakhmatov e Vrudhula; $E_{R V}$ é o erro gerado pela simulação do modelo de Difusão de Rakhmatov e Vrudhula em comparação com os dados obtidos da plataforma de testes, em \%.

Tabela 3. Comparação dos resultados do Modelo Autorregressivo e do Modelo de Difusão de Rakhmatov-Vrudhula.

\begin{tabular}{|c|c|c|c|c|c|}
\hline $\begin{array}{c}I \\
(\mathrm{~mA})\end{array}$ & $\begin{array}{c}T V_{m} \\
(\mathrm{~min})\end{array}$ & $\begin{array}{l}\mathrm{TV}_{\mathrm{SAR}} \\
(\mathrm{min})\end{array}$ & $\begin{array}{l}E_{A R} \\
(\%)\end{array}$ & $\begin{array}{l}\mathrm{TV}_{\mathrm{SRV}} \\
(\mathrm{min})\end{array}$ & $\begin{array}{l}E_{\mathrm{RV}} \\
(\%)\end{array}$ \\
\hline 200 & 136,66 & 136,66 & 0 & 143,00 & 4,43 \\
\hline 350 & 77,95 & 77,95 & 0 & 79,89 & 2,49 \\
\hline 500 & 49,38 & 51,90 & 4,86 & 54,67 & 10,71 \\
\hline 650 & 40,55 & 38,93 & 4,15 & 41,00 & 1,16 \\
\hline 800 & 28,8 & 31,41 & 8,31 & 32,56 & 13,05 \\
\hline 950 & 26,08 & 26,32 & 0,92 & 26,67 & 2,26 \\
\hline \multicolumn{2}{|c|}{ Erro Médio: } & & 3,04 & & 5,68 \\
\hline
\end{tabular}

\section{Conclusões}

Com isso, conclui-se que o modelo $\mathrm{AR}$ ao ser aplicado a um conjunto de dados obtido a partir da plataforma de teste possui resultados satisfatórios, pois apresentou um erro médio de $3,04 \%$, erro este, $2,64 \%$, menor do que o erro médio apresentado pelo Modelo de Difusão de Rakhmatov e Vrudhula mostrando, assim, sua eficiência. Além disto, o modelo AR mostrou-se um modelo simples de ser entendido, fácil de ser implementado e que traz bons resultados. Com este estudo, pode-se perceber que voltar a atenção para a teoria da Identificação de Sistemas, na modelagem da predição do tempo de vida de baterias, é algo que se mostra interessante e abre caminho para a utilização desta para a modelagem dos mais diversos sistemas, pois a Identificação de Sistemas se mostra mais interessante do ponto de vista econômico, se comparada com a experimentação física, gerando menos gastos, além de mostrar-se mais simples quanto ao critério de entendimento das estruturas dos modelos e implementação dos mesmos, exigindo, também, menor tempo computacional.

\section{Agradecimentos}

Agradecemos a Universidade Regional do Noroeste do Estado do Rio Grande do Sul - UNIJUÍ por ceder o espaço para o desenvolvimento do trabalho, pela bolsa de auxílio para o desenvolvimento dos estudos no Curso de Pós-Graduação Stricto Sensu em Modelagem Matemática (Mestrado em Modelagem Matemática), ao Mestrado em Modelagem Matemática pela aprendizagem e oportunidade de conhecer e desenvolver estudos com estas novas teorias em matemática e ao Grupo de Automação Industrial e Controle - GAIC pela utilização da plataforma de testes. 


\section{Referências Bibliográficas}

Aguirre, L. A. (2004). Introdução à identificação de sistemas: técnicas lineares e não-lineares aplicadas a sistemas reais. Editora UFMG, $2^{\mathrm{a}}$ edição, Belo Horizonte - MG.

Anton, H. and Rorres, C. (2001). Álgebra Linear com Aplicações. $8^{\text {a }}$ Edição, Bookman, Porto Alegre RS.

Chen, M. and Rincón-Mora, G. A. (2006). Accurate electrical battery model capable of predicting runtime and I-V performance. IEEE Transactions on Energy Conversion, 21, $\mathrm{n}^{\mathrm{o}}$ 2. DOI: 10.1109/TEC.2006.874229

Chiasserini, C. and Rao, R. (1999). A Model for Battery Pulsed Discharge with Recovery Effect. Proceedings of the Wireless Communications and Networking Conference, pp. 636-639.

Jongerden, M. and Haverkort, B. (2008). Battery Modeling. Technical Report in Faculty Electrical Engineering, Mathematics and Computer Science.

Lahiri, K.; Raguhnathan, A.; Dey, S. and Panigrahi, D. (2002). Battery-driven system design: a new frontier in low power design. Chemical Engineering Science.

Martin, T. L. (1999). Balancing Batteries, Power, and Performance: System Issues in CPU SpeedSetting for Mobile Computing. Master's Thesis, Carnegie Mellon University.

Morettin, P. A. and Toloi C. M. C. (1987). Previsão de séries temporais. Atual, $2^{\mathrm{a}}$ Edição, São Paulo $-\mathrm{SP}$.

Oliveira, A. V. (2012). Análise comparativa de metodologias de estimação de parâmetros aplicada a modelos analíticos utilizados na predição do tempo de vida de uma bateria. Dissertação de Mestrado, Universidade Regional do Noroeste do Estado do Rio Grande do Sul, Ijuí - RS.

Rakhmatov, D. and Vrudhula, S. (2001). An Analytical High-Level Battery Model for Use in Energy Management of Portable Electronic Systems. Proceedings of the 2001 IEEE/ACM International Conference on Computer-Aided Design, pp. 488-493.

Romio, L. C. (2013). Modelagem Matemática da Predição do Tempo de Vida de Baterias Utilizando Identificação de Sistemas. Dissertação de Mestrado, Universidade Regional do Noroeste do Estado do Rio Grande do Sul, Ijuí - RS.

Sausen, P. (2008). Gerenciamento Integrado de Energia e Controle de Topologia em Redes de Sensores Sem Fio. Tese de Doutorado, Universidade Federal de Campina Grande, UFCG, Campina Grande - PB.

Schneider K. (2011). Modelos analíticos na predição do tempo de vida de baterias utilizadas em dispositivos móveis. Dissertação de Mestrado, Universidade Regional do Noroeste do Estado do Rio Grande do Sul, Ijuí - RS. 